EXTENDED REPORT

\title{
Osteocyte viability with glucocorticoid treatment: relation to histomorphometry
}

\author{
P N Sambrook, D R Hughes, A E Nelson, B G Robinson, R S Mason
}

See end of article for

authors' affiliations

Correspondence to: Professor P N Sambrook Sydney University, Royal North Shore Hospital St Leonards Sydney, Australia 2065; sambrook@med.usyd. edu.au

Accepted 12 May 2003
Ann Rheum Dis 2003;62:1215-1217. doi: 10.1136/ard.2003.008839

\begin{abstract}
Background: Glucocorticoid induced osteoporosis is a common clinical problem. Objective: To determine the pathophysiology of glucocorticoid induced osteoarthritis at the organ level. Methods: Iliac crest biopsy specimens were obtained from nine patients receiving prednisone treatment for rheumatoid arthritis. Osteocyte viability and histomorphometric indices were assessed.

Results: Compared with controls, glucocorticoid treated subjects had reduced trabecular thickness and increased erosion. The number of viable osteocytes was significantly decreased in glucocorticoid treated patients compared with controls.

Conclusion: The impaired bone formation, increased erosion and, importantly, loss of viable osteocytes are all likely to contribute to the increased risk of fracture in these patients.
\end{abstract}

G lucocorticoid induced osteoporosis is common owing to the effects of glucocorticoids on bone metabolism through multiple pathways, influencing both bone formation and resorption. ${ }^{1}$ For the most part, the decreased bone formation is due to direct effects on cells of the osteoblastic lineage, although indirect effects related to sex steroid production are also important. It has been suggested that enhanced osteoctye apoptosis is as an important mechanism of glucocorticoid osteoporosis ${ }^{23}$ In animal models, glucocorticoids have been shown to reduce the formation rate of osteoblasts and osteoclasts and cause the earlier death of osteoblasts. ${ }^{4}$ The promotion of apoptosis in osteocytes and in osteoblasts by glucocorticoids is a possible mechanism for the associated rapid increase in fracture susceptibility. As well as undergoing cell death by apoptosis, osteocytes may undergo necrosis when bone age exceeds the upper limit of the lifespan of osteocytes, ${ }^{5-7}$ although the effect of glucocorticoids on osteocyte lifespan independent of apoptosis is unknown.

The direct inhibitory effects of glucocorticoids on bone formation have also been documented in histomorphometric studies, $^{8-10}$ although no studies have correlated osteocyte viability with histomorphometric changes. Osteocytes have two possible fates, remaining in bone until replacement by remodelling or dying through apoptosis, leaving an empty lacuna. ${ }^{11}$ Most studies of apoptosis have used transferase mediated biotin dUTP nick end labelling (called the TUNEL reaction) to assess cell death during the limited period of time during which this process is taking place. ${ }^{5}$ Assessment of replacement by modelling can be made by determining osteocyte density. ${ }^{311}$ Assessment of the viability of osteocytes is normally monitored by their lactate dehydrogenase (LDH) activity $^{12}{ }^{13}$ and reduced viability is likely to reflect incipient apoptosis.

In this study we assessed osteocyte viability and histomorphometric indices in patients with rheumatoid arthritis treated with glucocorticoids.

\section{SUBJECTS AND METHODS}

\section{Patients with rheumatoid arthritis}

Iliac crest biopsy specimens were obtained from nine women, aged 61-79 years, who were receiving prednisone treatment (mean daily dose $6.8 \mathrm{mg}$, range 4-12.5) for rheumatoid arthritis. Before the biopsy, patients received demeclocycline
$300 \mathrm{mg}$ three times daily or tetracycline $500 \mathrm{mg}$ three times daily (for 2 days on, 12 days off, 2 days on). Biopsy specimens were taken five days after the last dose. Separate biopsy specimens for histomorphometry and osteocyte viability assessment were obtained from each patient. These specimens were put into $70 \%$ ethanol for histomorphometry or normal saline for osteocyte viability processing, respectively.

\section{Controls}

Lateral medial plateau (one female, four male), femoral condyle (one male), or femoral head (one male) biopsy specimens were obtained from patients, aged 55-79 years, who had had knee or hip replacements for osteoarthritis. These patients did not take tetracyclines before the operation. The biopsy specimens were fixed in $70 \%$ ethanol and processed as histomorphometry controls.

Femoral heads were obtained from eight women aged 7295 years. They were accepted for the study only if the operations were carried out on the day after admission to hospital for subcapital fracture of the femoral neck after minimal trauma or earlier. After removal, the femoral heads were immersed in normal saline. They were kept at $0-4^{\circ} \mathrm{C}$ until processing that was started within 24 hours of the operation. The femoral head bone was processed for osteocyte viability controls.

Ethical approval for the study was given by the relevant human research ethics committee and informed consent was obtained before biopsy

\section{Bone histomorphometry}

After dehydration in graded ethanols, biopsy specimens were embedded in methyl methacrylate. Serial sections were cut at two levels. They were stained by a modified Masson trichrome method ${ }^{14}$ for measurement of the following variables: bone volume/total volume (BV/TV, \%), trabecular thickness ( $\mathrm{Tb} \mathrm{Th}, \mu \mathrm{m})$, trabecular number ( $\mathrm{TB} \mathrm{No} / \mathrm{mm}$ ), trabecular spacing ( $\mathrm{Tb} \mathrm{Sp}, \mu \mathrm{m})$, eroded surface/bone surface (ES/BS, \%), osteoid surface/bone surface (OS/BS, \%), osteoid thickness (O Th, $\mu \mathrm{m}$ ), osteoblast surface/bone surface (Ob S/ BS, \%). An acid phosphatase method (naphthol AS-BI phosphate-hexazonium pararosaline method), ${ }^{15}$ with $28 \mathrm{mg} /$ $10 \mathrm{ml}$ of sodium tartrate added to the incubation solution, was used to measure the surface under the osteoclasts (Oc S/ BS, \%). Sections from biopsy specimens of patients with 
rheumatoid arthritis were left unstained for evaluation of tetracycline labelled surface (LS/BS) under ultraviolet light. All variables were measured with the semiautomatic OsteoMeasure Histomorphometry video system (Osteometrics, Atlanta, USA), which uses the ASMBR Committee nomenclature. ${ }^{16}$

\section{Osteocyte viability assessment}

Three to four $300 \mu \mathrm{m}$ thick sections of biopsy specimens from patients with rheumatoid arthritis were sawn with a Leica 1600 saw microtome (Leica, Sydney) within 1.5 hours of biopsy. Four $300 \mu \mathrm{m}$ thick sections of femoral head bone were sawn perpendicular to the ligamentum teres about $12 \mathrm{~mm}$ from its insertion point (the fovea) with a Microslice 2 precision saw (Metals Research, Cambridge, England). All sections were decalcified in 10\% EDTA in Tris buffer $0.05 \mathrm{M}$, $\mathrm{pH} 7.0,6^{\circ} \mathrm{C}$ for 16 hours. After rinsing thoroughly in Tris buffer, histochemical staining was undertaken using a modification of the method of Wong et al. ${ }^{6}$ Briefly, $300 \mu \mathrm{m}$ sections were allowed to react in $0.1 \%$ magnesium chloride in Tris buffer $0.05 \mathrm{M}, \mathrm{pH} 7.0,50 \mathrm{mmol} / \mathrm{l}$ lithium lactate (Sigma), $0.18 \mathrm{mg} / \mathrm{l}$ nitroblue tetrazolium (Sigma), and $0.7 \mathrm{mg} / \mathrm{l}$ nicotinamide adenine dinucleotide (Sigma) for four hours at $37^{\circ} \mathrm{C}$. All sections were fixed in $70 \%$ ethanol. Sawn surfaces of bone trabeculae in sections with marrow removed were cleaned with $0.1 \% \mathrm{HCl}$ for $2 \times 2$ minutes to avoid surface artefact. After rinsing in water, sections were stained with methyl green for 15 minutes. After thorough rinsing with water, sections were rolled flat after covering with pieces of polyethylene plastic, clamped between glass slides, oven dried at $60^{\circ} \mathrm{C}$ for at least three hours, and mounted in Eukitt.

Viable osteocytes were identified by the presence of dark blue formazan granules in the cytoplasm. Non-viable osteocytes were identified by methyl green stained nuclei and the absence of blue granules in the cytoplasm. Empty lacunae were identified by their lacunar walls with phase contrast that was produced by flipping out the condenser lens of the microscope. Osteocytes and lacunae were located by focusing through several planes within $300 \mu \mathrm{m}$ thick, translucent sections and counted using the OsteoMeasure histomorphometry video system (Osteometrics, Atlanta, USA) at an objective magnification of 20. Statistical analysis was performed using a one tailed Student's $t$ test.

Marrow viability was assessed qualitatively. If the bone marrow across the entire section showed uniform, intense staining for LDH activity and no areas of necrosis were seen, then the bone marrow at the time of biopsy or fracture was considered viable. If there was no intense LDH staining or heterogeneity in staining across the section, then the sample was rejected. LDH staining is the darkest of dark blue and there is no doubt at all if areas or significant groups of cells have died. One would expect isolated dead cells to occur throughout the tissue. All bone marrow sections used to control for necrosis in biopsy and hip fracture sections were assessed as viable. All samples were accepted as assessable for osteocyte viability for the biopsy specimens but a number of femoral head samples from additional patients with hip fracture were rejected.

\section{RESULTS}

Table 1 shows the histomorphometric indices and table 2 the osteocyte viability. Compared with osteoarthritic controls and normative data for this age group from transiliac bone biopsy specimens, ${ }^{17}$ glucocorticoid treated subjects had reduced trabecular thickness $(\mathrm{p}<0.01)$ and an increase of eroded surfaces $(\mathrm{p}<0.05)$ (table 1$)$.

The number of viable cells was significantly decreased in glucocorticoid treated patients compared with controls $(\mathrm{p}<0.05)$ (table 2$)$. The percentage of viable ( $\mathrm{LDH}$ positive)
Table 1 Histomorphometric indices (mean (SD))

\begin{tabular}{|c|c|c|c|}
\hline & $\begin{array}{l}\text { Patients receiving } \\
\text { GCs }(n=9)\end{array}$ & $\begin{array}{l}\text { Controls (hip } \\
\text { and knee, } n=7 \text { ) }\end{array}$ & $\begin{array}{l}\text { Controls, } \\
65-74 \text { years } \\
\text { Recker et al }{ }^{7}\end{array}$ \\
\hline BV/TV (\%) & $19.3(4.4)$ & $28.1(5.1)$ & $19.6(5.6)$ \\
\hline $\mathrm{Tb}$ Th $(\mu \mathrm{m})$ & $82.8(12.8)^{* *}$ & $118.2(18.8)$ & $131.3(28.1$ \\
\hline $\mathrm{Tb} \mathrm{No} / \mathrm{mm}$ & $2.30(0.30)$ & $2.4(0.34)$ & $1.5(0.29)$ \\
\hline $\mathrm{Tb} S p(\mu \mathrm{m})$ & $357.2(72.2)$ & $307.5(53.1)$ & $690.5(178.0)$ \\
\hline ES/BS (\%) & $7.1(4.4)^{\star}$ & 2.5 (1.4) & $3.7(1.7)$ \\
\hline Oc S/BS (\%) & 1.25 (1.37) & $0.20(0.17)$ & $0.6(0.7)$ \\
\hline OS/BS (\%) & $5.8(3.4)$ & 4.7 (3.4) & $14.0(6.6)$ \\
\hline O Th $(\mu \mathrm{m})$ & $5.4(1.6)$ & $6.0(1.74)$ & $8.3(2.0)$ \\
\hline $\mathrm{Ob} \mathrm{S} / \mathrm{BS}$ & $0.97(1.0)$ & $0.29(0.39)$ & $3.11(2.8)$ \\
\hline LS/BS & $4.3(3.9)$ & - & 3.4 (3.1) \\
\hline
\end{tabular}

Significantly different from both sets of controls: ${ }^{*} p<0.05 ;{ }^{* *} p<0.01$. $\mathrm{BV} / \mathrm{TV}$, bone volume/total volume; Tb Th, trabecular thickness; TB No, trabecular number; Tb Sp, trabecular spacing; ES/BS, eroded surface/ bone surface; Oc S/BS, osteoclast surface/bone surface; OS/BS, osteoid surface/bone surface; O Th, osteoid thickness; Ob S/BS, osteoblast surface/bone surface; LS/BS, labelled surface/bone surface.

\begin{tabular}{lcc} 
Table 2 & Osteocyte viability (mean (SD)) \\
\hline & $\begin{array}{l}\text { Patients receiving } \\
\text { GCs }(\mathbf{n}=9)\end{array}$ & $\begin{array}{l}\text { Hip fracture controls } \\
(\mathbf{n}=8)\end{array}$ \\
\hline Viable & $620(93)^{*}$ & $743(135)$ \\
Non-viable & $34(45)$ & $15(11)$ \\
Empty lacunae & $52(49)$ & $36(33)$ \\
Cells (n) & $706(107)$ & $795(150)$ \\
Viable (\%) & $88(8)^{*}$ & $94(4)$ \\
Non-viable (\%) & $5(6)$ & $2(2)$ \\
Empty lacunae (\%) & $7(5)$ & $4(4)$ \\
Non-viable+empty & $128^{*}$ & $6(4)$ \\
lacunae (\%) & & \\
\hline \multicolumn{2}{l}{ Significantly different from control group: ${ }^{*} \mathrm{p}<0.05}$.
\end{tabular}

osteocytes in glucocorticoid treated patients was based on a total of 705 osteocytes and for hip fracture patients on a total of 795 osteocytes and empty lacunae in eight randomly selected fields from one section for each patient. Similarly, the percentage of non-viable and empty lacunae was significantly increased in glucocorticoid treated patients. In glucocorticoid treated patients, there was a significant correlation between the number of viable cells and OS/BS $\left(r_{\mathrm{s}}=0.74, \mathrm{p}<0.05\right)$.

\section{DISCUSSION}

Although osteocytes reside in lacunae within the mineralised matrix of bone, they are not considered inactive cells, but rather they probably have a role in transduction of signals of mechanical loading, thereby acting as the mechanosensors in bone. ${ }^{18}$ Osteocytes are long lived cells, ${ }^{5}$ but some of them die as shown by the presence of empty lacunae or lack of enzyme (LDH) activity. ${ }^{12}$ Decreased osteocyte viability and increased apoptosis will be reflected in decreased osteocyte density, which has been related to age and bone formation rate in healthy (non-glucocorticoid treated) postmenopausal women. ${ }^{71}$ Although enhanced osteocyte apoptosis has been implicated as an important mechanism of osteoporosis due to oestrogen deficiency, ${ }^{19}$ and in animal models of glucocorticoid induced osteoporosis, ${ }^{4}$ there is limited evidence for this in humans. ${ }^{3}{ }^{4}$ These authors identified apoptotic osteoblasts and osteocytes in transiliac biopsy specimens from two patients with glucocorticoid induced osteoporosis, in contrast with biopsy specimens from normal controls, in which no apoptotic cells were detected, ${ }^{4}$ and apoptotic osteocytes in the femoral heads of patients with glucocorticoid induced 
osteonecrosis. ${ }^{3}$ We found the number of viable osteocytes was significantly decreased in glucocorticoid treated patients compared with controls, consistent with this hypothesis.

In the study presented here the numbers of patients investigated are greater, but the controls are not ideal. For practical reasons, relating to the difficulty of ethically justifying an iliac crest biopsy in normal subjects, the control biopsy specimens were from the femoral heads rather than the iliac crest. Although there have been reported differences in osteocyte viability in the spine compared with the hip, ${ }^{20}$ the controls for osteocyte viability were all female, but somewhat older than the glucocorticoid treated group, which would be likely to decrease the chance of finding viable osteocytes in that group as viability has been reported to decrease with age. ${ }^{6}$ These control patients had also sustained a hip fracture, which would also not be expected to enhance osteocyte viability in the removed femoral heads. Moreover, our control data are consistent with the percentage total occupied lacunae at age 80 reported by Qui et al of about $90 \%$. Moreover, Qui et al recognised that some stained osteocytes might have recently died by apoptosis. ${ }^{7}$ In our study we quantified the percentage of dead but stainable osteocytes by methyl green nuclei staining and lack of LDH product. Of the $12 \%$ non-viable cells and empty lacunae group in glucocorticoid treated patients, $5 \%$ were recently dead. In contrast, in the controls $2 \%$ of the $6 \%$ were recently dead. These findings suggest that glucocorticoid treated patients have an increased number of non-viable (recently dead) osteocytes.

The controls used for histomorphometry were better age matched but were predominantly male. For this reason, data from age matched female controls from the study of Recker et al, ${ }^{17}$ using the Osteomeasure system, were also considered. The trabecular thickness and eroded surface were similar to those of the current controls and different from the glucocorticoid treated group.

Reduced bone formation is characteristic of glucocorticoid induced osteoporosis. ${ }^{21}$ The effect of glucocorticoids on osteoclastic activity is rather more variable. Osteoclastic activity is mostly increased in this condition, ${ }^{19}$ though not in all studies. ${ }^{4}$ Glucocorticoids have been reported to reduce osteoclastogenesis in vitro, ${ }^{4}$ though possibly only at very high doses, ${ }^{22}$ and more consistently to increase osteoclast lifespan. ${ }^{19} 22$ The increased osteoclastic activity in the patients reported here might be explained by an increase in osteoclast lifespan in the presence of glucocorticoids.

The clinical significance of the observed decrease in osteocyte viability in patients treated with glucocorticoids is unclear at this time. Nevertheless, it is reasonable to speculate that decreased osteocyte viability might diminish bone ability to sense mechanical load and so lead to reduced bone mass. The observed correlation between osteocyte viability and osteoid surface may support this proposal. Decreased osteocyte viability might also decrease the ability to sense or repair microdamage and so contribute to an increased propensity to fracture for a given bone mineral density. Loss of osteocyte viability in large areas might also lead to so-called avascular osteonecrosis. ${ }^{21}$

\section{ACKNOWLEDGEMENTS}

Erin Martin is acknowledged for technical assistance.

\section{Authors' affiliations}

P N Sambrook, Institute of Bone and Joint Research, Royal North Shore Hospital, University of Sydney, Australia

D R Hughes, Department of Endocrinology and Metabolism, Concord Hospital, Australia

A E Nelson, B G Robinson, Department of Cancer Genetics, Kolling Institute for Medical Research, Royal North Shore Hospital and Department of Physiology, Institute for Biomedical Research, University of Sydney, Australia

R S Mason, Department of Physiology and Institute for Biomedical Research, University of Sydney, Australia

\section{REFERENCES}

1 Sambrook P, Lane NE. Corticosteroid osteoporosis, Balliere's Best Practice and Research. Clin Rheumatol 2001;15:401-13.

2 Canalis E. Mechanisms of glucocorticoid action in bone: implications to glucocorticoid induced osteoporosis. J Clin Endocrinol Metab 1996;81:3441-7.

3 Weinstein RS, Nicholas RW, Manolagos SC. Apoptosis of osteocytes in glucocorticoid induced osteonecrosis of the hip. J Clin Endocrinol Metab 2000;85:2907-12

4 Weinstein RS, Jilka RL, Parfitt AM, Manolagos SC. Inhibition of osteoblastogenesis and promotion of apoptosis of osteoblasts and osteocytes by glucocorticoids. J Clin Invest 1998;102:274-82.

5 Frost H. In vivo osteoctye death, 1960;42:138-43.

6 Wong SYP, Kariks J, Evans RA, Dunstan CR, Hills E. Effect of age on bone composition and viability in the femoral head. J Bone Joint Surg Am 1985;67:274-83

7 Qui S, Rao DS, Palnitkar S, Parfitt AM. Age and distance from the surface but not menopause reduce osteocyte density in human cancellous bone. Bone 2002;31:313-18.

8 Bressot C, Meunier PJ, Chapuy MC, Lejeune E, Edouard C, Darby AJ. Histomorphometric profile, pathophysiology and reversibility of corticosteroid induced osteoporosis. Metab Bone Dis Rel Res 1979;1:303-11.

9 Dalle Carbonare L, Arlot ME, Chavassieux PM, Roux JP, Portero NR, Meunier PJ. Comparison of trabecular bone microarchitecture and remodeling in glucocorticoid-induced and postmenopausal osteoporosis. J Bone Miner Res 2001; 16:97-103.

10 Chappard D, Legrand E, Basle MF, Fromont P, Racineux JL, Rebel A, et al. Altered trabecular architecture induced by glucocorticoids: a bone histomorphometric study. J Bone Miner Res 1996;11:676-85.

11 Qui S, Rao DS, Palnitkar S, Parfitt AM. Relationship between osteocyte density and bone formation rate in human cancellous bone. Bone 2002;31:709-11.

12 Wong SYP, Dunstan CR, Evans RA, Hills E. Determination of bone viability: a histochemical method of identification of lactate dehydrogenase activity in osteocytes in fresh calcified and decalcified sections of human bone. Pathology 1982; 14:439-42.

13 Noble BS, Stevens H, Loveridge N, Reeve J. Identification of apoptotic changes in osteocytes in normal and pathological human bone. Bone 1997; 20:273-82.

14 Luna LG. In: Luna LG, ed. Manual of histological staining methods of the Armed Forces Institute of Pathology, 3rd ed. New York: McGraw-Hill, 1968.

15 Evans RA, Dunstan CR, Baylink DJ. Histochemical identification of osteoclasts in undecalcified sections of human bone. Mineral Electrolyte Metabolism 1979;2:179-85.

16 Parfitt AM, Drezner MK, Glorieux F, Kanis JA, Malluche H, Meunier PJ, et al. Bone histomorphometry: standardization of nomenclature, symbols, and units. $J$ Bone Miner Res 1987; 2:595-610.

17 Recker RR, Kimmel DB, Parfitt AM, Davies KM, Keshawarz N, Hinders S. Static and tetracycline-based bone histomorphometric data from 34 normal postmenopausal females. J Bone Miner Res 1988;3:133-44.

18 Klein-Nulend J, Van der Plas A, Senmeins CM, Ajubi NE, Frangos JA, Niiweide PJ et al. Sensitivity of osteoctyes to biochemical stress in vitro. FASEB J 1995;59:441-5.

19 Weinstein RS, Chen JR, Powers CC, Stewart SA, Landes RD, Bellido T, et al. Promotion of osteoclast survival and antagonism of bisphosphonate-induced osteoclast apoptosis by glucocorticoids. J Clin Invest 2002;109:1041-8.

20 Dunstan CR, Somers NM, Evans RA. Osteocyte cell death and hip fracture. Calcif Tiss Int 1993;53:S113-17

21 Dempster D. A perspective: bone histomorphometry in glucocorticoid-induced osteoporosis. J Bone Min Res 1989:4:137-41.

22 Sivagurunathan S, Muir MM, Seale JP, Mason RS. Direct effects of dexamethasone on osteoclasts and precursors [abstract]. Aust NZ: Orthopaedic Research Society, 2002 (8th Annual Scientific Meeting). 